NSL 09642

\title{
Influence of barium on rectification in rat neocortical neurons
}

\author{
Bernd Sutor and John J. Hablitz \\ Department of Physiology, University of Munich. Munich (FRG) and Neurobiology Research Center, University of Alabama at Birmingham. \\ Birmingham. AL 35294 (USA)
}

(Received 6 February 1993; Revised version recieved 10 April 1993; Accepted 10 April 1993)

Key words: Neocortex; Potassium current; Inward rectification; Barium

\begin{abstract}
The effect of low concentrations of barium on the membrane properties of rat neocortical neurons was studied in vitro. Potassium currents were examined using single-electrode current- and voltage-clamp techniques. Neurons responded to bath application of barium (10-100 $\mu \mathrm{M}$ ) with a membrane depolarization associated with an increase in input resistance. Under voltage clamp conditions, an inward shift in holding current was observed. The effects of barium were rapidly reversible upon washing and persisted in the presence of TTX. The equilibrium potential for the barium-induced inward current was near $-110 \mathrm{mV}$, suggesting that barium inhibited a tonically active potassium conductance. Measurements of current voltage relationships indicated an inward rectification of this conductance between -50 and $-130 \mathrm{mV}$. These results provide strong evidence that barium blocks a persistent potassium 'leak' current in neocortical neurons that contributes to the resting potential of these cells.
\end{abstract}

Neocortical neurons in vitro display both inward and outward rectification $[6,14,17]$. The time-dependent depolarizing inward rectification of rodent neocortical neurons is due to activation of a low-voltage activated, transient calcium current while a time-independent rectification was attributed to a persistent sodium current $[4,17]$. However, the nature of the ionic current responsible for hyperpolarizing inward rectification in neocortical neurons has not been firmly established. Anomalous rectification is the term used to describe the original observation by Katz [7] that hyperpolarization of frog muscle fibers was associated with an increase in membrane conductance. Subsequently, two types of anomalous rectifiers have been recognized. The first type is mediated by an increase in potassium conductance and has relatively fast activation kinetics over a voltage-range which is dependent on the difference between the membrane potential and the potassium equilibrium potential. This type of rectifier is blocked by extracellular application of barium $[3,5,7,8]$. The second type of rectifier current has slower kinetics, is barium insensitive, blocked by cesium, and permeable to both sodium and potassium ions. This rectifier plays an important role in regulation of cardiac

Correspondence: J.J. Hablitz, Neurobiology Research Center, University of Alabama at Birmingham, 1719 Sixth Avenue South. Birmingham, AL 35294-0021. USA. Fax: (1) (205) 934-6571. excitability [1] and has been identified in many central neurons $[6,9,10,13]$.

Inward rectification in neocortical neurons is manifested in two ways. First, during passage of hyperpolarizing current pulses, voltage responses reach a peak and then decline or 'sag' back towards the resting level [15]. Second, plots of amplitudes of the steady-state voltage levels reached during hyperpolarizing current pulses of varying amplitude show a reduction in slope resistance [17]. Under voltage-clamp conditions, no time dependence of the hyperpolarizing inward rectification was observed [17]. Hwa and Avoli [6] have reported that barium reduced both the sag and steady-state rectification in neocortical neurons. However, the concentrations of barium employed were much higher than those originally reported to be effective in blocking the inward rectifier [5]. We now report that barium, at concentrations of $10100 \mu \mathrm{M}$. blocks an inwardly rectifying potassium conductance active at rest in layer II-III rat neocortical neurons. A preliminary account of this work has appeared in abstract form [16].

Layer II -III neurons were studied in an in vitro brain slice preparation as described previously [17]. Male Wistar rats $(120-180 \mathrm{~g})$ were decapitated under ether anesthesia. For recording, the slices were held between two pieces of nylon mesh and submerged in oxygenated saline maintained at a temperature of $31-32^{\circ} \mathrm{C}$.

The saline had the following composition (in $\mathrm{mM}$ ): 
$\mathrm{NaCl} 124, \mathrm{KCl} 3.0, \mathrm{NaH}_{2} \mathrm{PO}_{4} 1.25, \mathrm{CaCl}_{2}, 2, \mathrm{MgCl}_{2}, 1.3$, $\mathrm{NaHCO}_{3} 25$ and glucose 10 . This saline was continuously gassed with $95 \% \mathrm{O}_{2}$ and $5 \% \mathrm{CO}_{2}$ to give a final $\mathrm{pH}$ of 7.4 .

Intracellular recordings were obtained from layer IIIII pyramidal neurons using electrodes filled with $3 \mathrm{M}$ $\mathrm{KCl}(60-90 \mathrm{M} \Omega$ ). Current- and voltage-clamp recordings were made using an npi SEC $1 \mathrm{~L}$ switching amplifier, as previously described [17]. Measurements of $I / V$ relationships were made prior to and $15 \mathrm{~min}$ after application of drugs to the perfusate. Membrane potential was continuously monitored on a chart recorder during an experiment. Barium $\left(\mathrm{BaCl}_{2}\right)$, muscarine, carbachol and TTX were added directly to the saline just prior to use.

Intracellular recordings were obtained from 33 neurons located in the superficial layers of the neocortex. These neurons had a resting membrane potential of $-81.6 \pm 5.2 \mathrm{mV}$ (mean \pm S.D.) and an input resistance of $33.1 \pm 19.3 \mathrm{M} \Omega$. Suprathreshold depolarizing current pulses evoked repetitive discharge of single spikes suggesting that these cells were regular-spiking neurons. The response to bath application of $30 \mu \mathrm{M}$ barium is shown in Fig. 1. Under current clamp conditions, barium produced a depolarization $(7.4 \pm 3.3 \mathrm{mV} ; n=10)$ and an
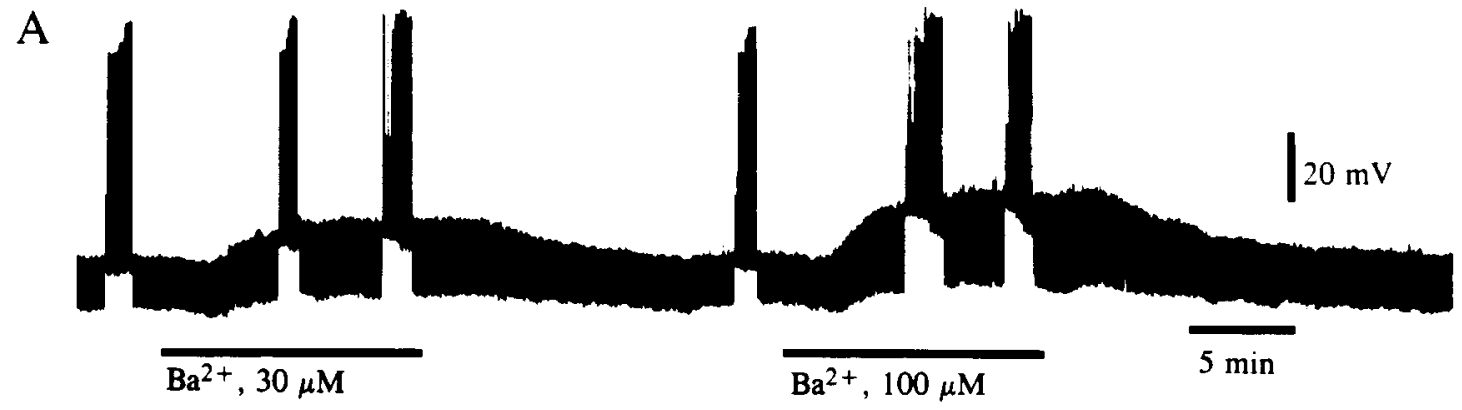

B control Barium, $30 \mu \mathrm{M}$

wash

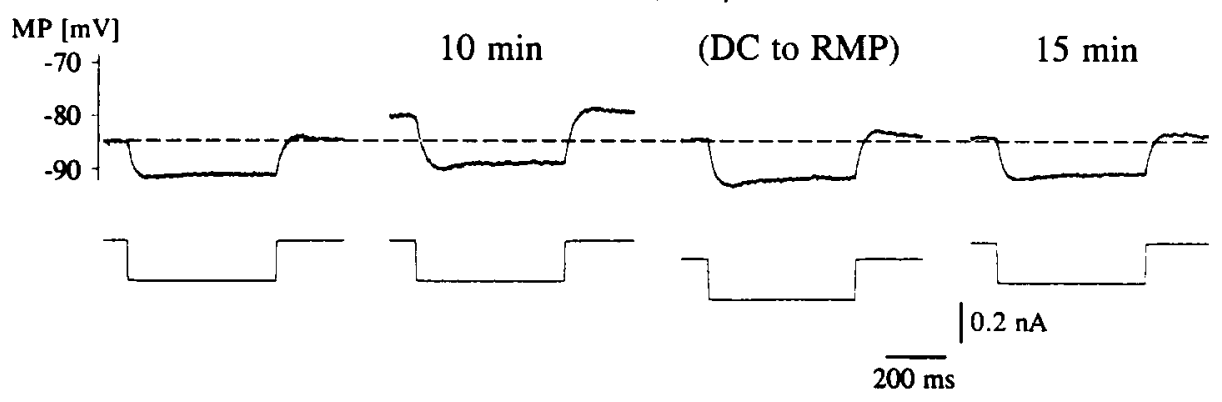

C

control

Barium, $30 \mu \mathrm{M}$

wash $10 \mathrm{~min}$

(DC to RMP)

$15 \min$

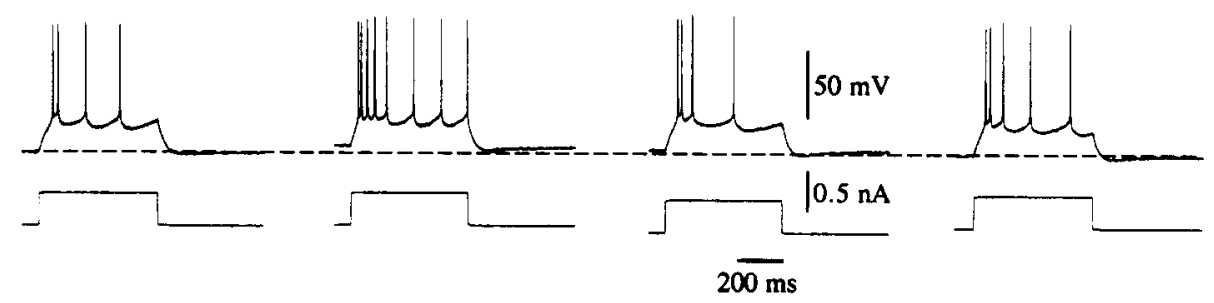

Fig. 1. A: low concentrations of $\mathrm{Ba}^{2+}$ depolarize rat neocortical neurons. Chart recordings show response to two concentrations of $\mathrm{Ba}^{2+}$ applied for the 15 min period indicated by the horizontal line. Up- and downward deflections represent the voltage responses to de- and hyperpolarizing current pulses. $\mathrm{B}$ : records from another neuron show that the $\mathrm{Ba}^{2+}$-induced increase in input resistance is independent of the membrane depolarization. Upper traces: voltage response to injected current. Lower traces: current monitor. $\mathrm{RMP}=-85 \mathrm{mV}$. $\mathrm{C}$ : effect of $\mathrm{Ba}^{2+}$ on repetitive firing properties of neocortical neurons. During the $\mathrm{Ba}^{2+}$-evoked depolarization the number of action potentials in response to a constant amplitude current pulse was increased. When the resting potential was returned to the control level, repetitive firing was no longer increased, suggesting $\mathrm{Ba}^{2+}$ was affecting excitability indirectly. RMP $=-79 \mathrm{mV}$. 
increase in input resistance $(53.9 \pm 24 \% ; n=10)$. When the concentration of barium was raised from 30 to 100 $\mu \mathrm{M}$, the amplitude of the membrane depolarization and resistance change increased. Since depolarization alone is known to cause an enhancement in input resistance of neocortical neurons [17], the changes in input resistance observed with barium could have been indirectly mediated. However, as shown in Fig. 1B, barium produced an increase in input resistance $(17.3 \pm 3.1 \% ; n=4)$ that was independent of the change in membrane potential. The number of action potentials produced by a constant amplitude depolarizing current pulse was increased after application of barium (Fig. 1C, panel 2), but this increase was not seen after the membrane potential was returned to control levels (Fig. 1C, panel 3). The effects of barium were fully reversible upon washing.

Under voltage clamp conditions (Fig. 2A), barium produced a dose-dependent inward shift in holding current. Analysis of plots of current amplitude versus barium concentration revealed an $\mathrm{EC}_{50}$ for barium's action of $60.3 \pm 17.8 \mu \mathrm{M}(n=5)$. As the holding potential was depolarized, the amplitude of the barium-induced current increased in a manner expected of a potassium dependent response (Fig. 2A).

Current-voltage relationships were determined by measuring current response at the end of a $500 \mathrm{~ms}$ step command, i.e. when the current change had reached a steady state (Fig. 2B,C). Barium produced a reversible
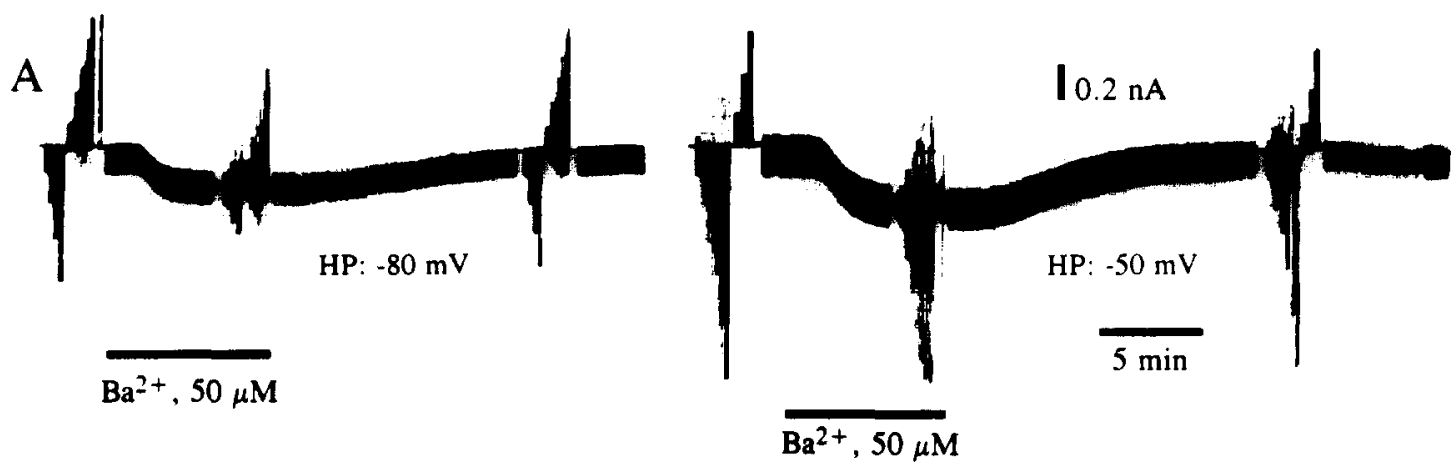

B control
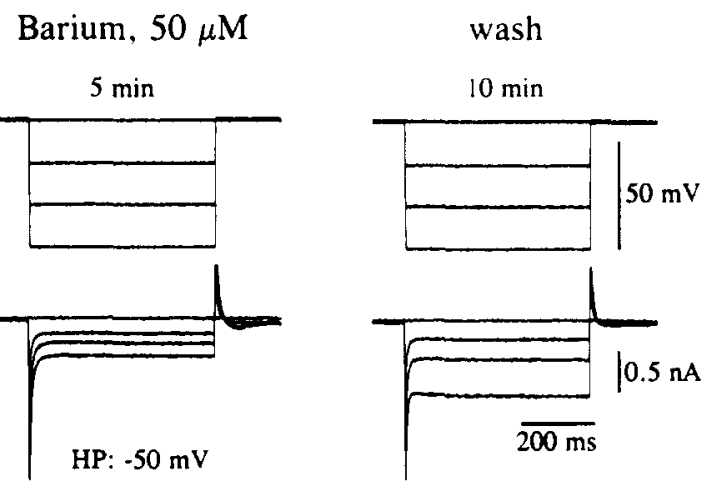

C
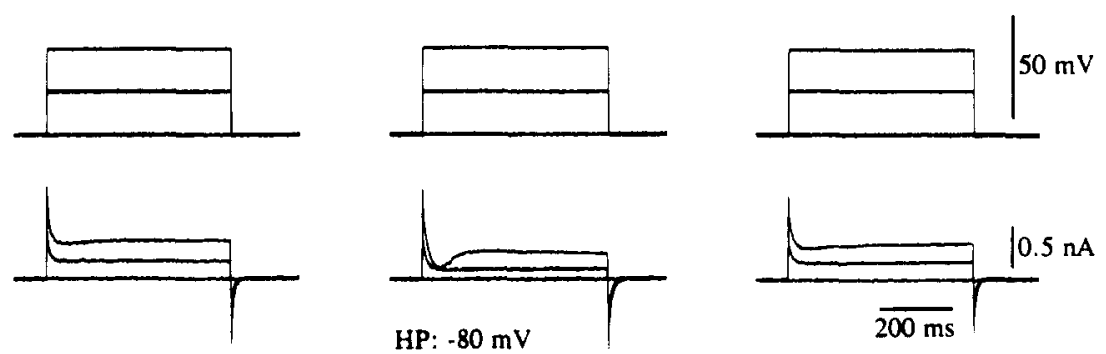

Fig. 2. Voltage-clamp analysis of $\mathrm{Ba}^{2+}$ effects on cortical neurons in the presence of $\mathrm{TTX}(0.6 \mu \mathrm{M})$. A: chart recorder tracings of membrane currents recorded at holding potentials of $-80 \mathrm{mV}$ (left) and $-50 \mathrm{mV}$ (right), respectively. $\mathrm{Ba}^{2+}(50 \mu \mathrm{M})$ was applied within the period indicated by the horizontal lines. $I / V$ curves were determined before during and following the application of $\mathrm{Ba}^{2+}$. B,C: measurement of $I / V$ curves in the absence and presence of $\mathrm{Ba}^{2+}(50 \mu \mathrm{M})$ using hyperpolarizing $(\mathrm{B}$, holding potential: $-50 \mathrm{mV}$ ) and depolarizing ( $\mathrm{C}$, holding potential: $-80 \mathrm{mV}$ ) voltage steps of 

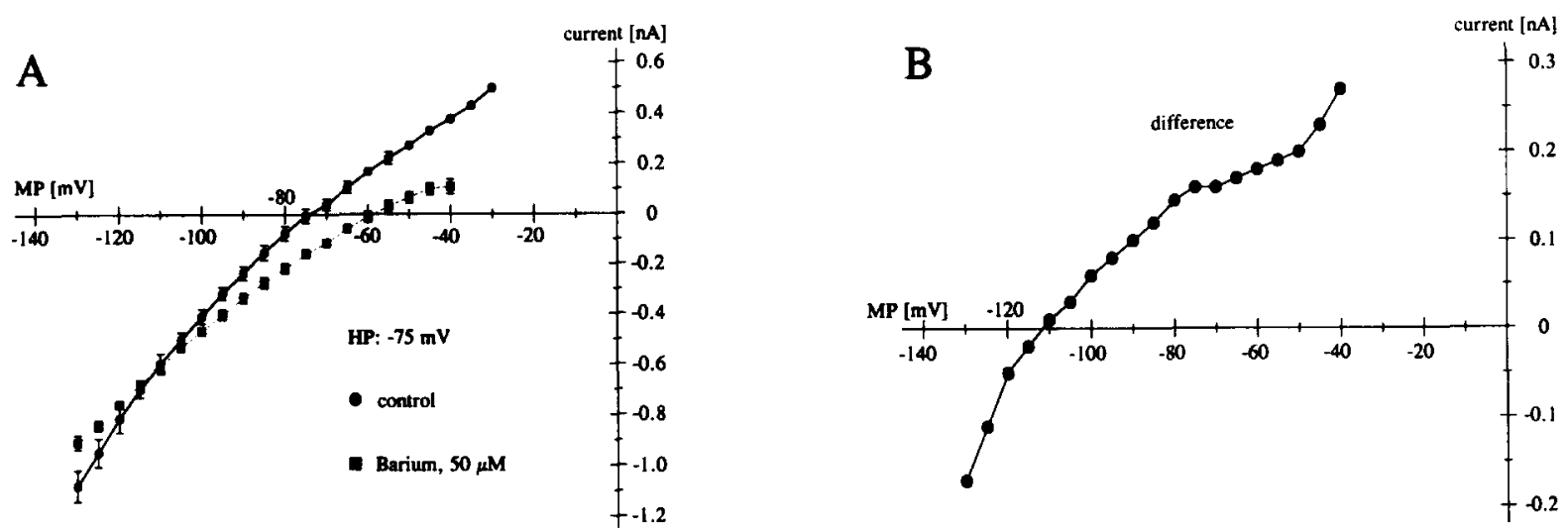

Fig. 3. A: current-voltage relationships obtained in the presence and absence of $50 \mu \mathrm{M} \mathrm{Ba}^{2+}$. The intersection of the $I / V$ curves occurred at $-109 \mathrm{mV}$. This is near the expected potassium equilibrium potential and is consistent with a potassium-mediated event. Each point represents mean S.D. from 3 neurons. B: difference curve obtained by subtracting the $I / V$ curve determined in the presence of $\mathrm{Ba}^{2+}$ from the control $I / V$ curve. The slope of the curve increases with hyperpolarization from -50 to $-130 \mathrm{mV}$, indicating inward rectification in this potential range.

reduction in the amplitude of the currents induced by both depolarizing and by hyperpolarizing voltage steps. Fig. 3A shows the $I / V$ curves before and during the application of barium at a concentration of $50 \mu \mathrm{M}$. The intersection of the $/ / V$ curves at $-110 \mathrm{mV}$ gives an estimate of the reversal potential for the response and indicates a value near the potassium equilibrium potential. Subtraction of the $I / V$ curve determined in the presence of barium from that obtained in the absence of the cation revealed that the potassium conductance inhibited by low concentrations of barium is inwardly rectifying within a potential range of -50 to $-130 \mathrm{mV}$ (Fig. 3C).

We observed a depolarizing action of low concentrations of barium that was associated with an increase in input resistance. These results are consistent with a reduction in a potassium conductance active at rest in neocortical neurons. The characteristics of this conductance, i.e., activation properties, kinetics and barium sensitivity, distinguish it from several previously described potassium currents in neocortical neurons.

An anomalous rectifier current has been described in both cat [13] and rat [6] neocortical neurons. In current clamp recordings it is manifest as a 'sag' of the membrane potential back towards resting levels during a hyperpolarizing current pulse. It is blocked by extracellular cesium ions and is insensitive [14] or weakly sensitive [6] to barium. Such 'sag' responses were observed during the current experiments (cf. Fig. 1B) but were not significantly affected by barium at the concentrations employed. It therefore appears that, in addition to a barium-insensitive, cesium-sensitive slow rectifier, neocortical neurons also possess a barium-sensitive rectifier that is tonically activated.

The current underlying the barium induced depolarization appeared to be potassium dependent. The ampli- tude of the inward current increased as the holding potential was made less negative and estimates of the reversal potential for the current yielded values near the potassium equilibrium potential. These characteristics clearly distinguish this response from an inwardly rectifying current described in a number of central neurons, termed $I_{\mathrm{Q}}$ or $I_{\mathrm{H}}$. This latter current has relatively slow kinetics and is a mixed $\mathrm{Na} / \mathrm{K}$ conductance with a reversal potential near $-45 \mathrm{mV}[9,13]$. The present findings resemble those seen with agonists such as muscarine and carbachol which affect the M-current [2]. However, neocortical neurons do not show the slow relaxations on offset of hyperpolarizing pulses that are characteristic of cells with robust M-currents. Moreover, barium was effective in a voltage range where $\mathrm{M}$-currents are not likely to be active. It is also improbable that barium is producing or enhancing an inward calcium current. Barium was added in the presence of $2 \mathrm{mM}$ extracellular calcium and is unlikely to cause a significantly increased inward current through calcium channels. Although barium is more permeant through L- and N-type calcium channels, it is equipermeant with calcium through $\mathrm{T}$ channels. The latter would be expected to be the only calcium channels active at the negative membrane potentials where barium was generally tested. Barium therefore appears to be affecting a potassium channel. Potassium currents are commonly modulated by a wide variety of neurotransmitters and intracellular second messengers [11]. Since the current described here is active at rest it could be a prime target of modulators which induce tonic changes in postsynaptic excitability.

This work was supported by Deutsche Forschungsgemeinschaft, SFB 220, TP A9 and National Institute of Neurological Disorders and Stroke grant NS18145. We 
thank R.C. Foehring for comments on an earlier version of this paper.

1 Brown, H. and DiFrancesco, D., Voltage-clamp investigations of membrane currents underlying pace-maker activity in rabbit sinoatrial node, J. Physiol., 308 (1980) 331-351.

2 Constanti, A., Adams. P.R. and Brown, D.A.. Why do barium ions imitate acetylcholine?, Brain Res., 206 (1981) 244-250.

3 Constanti, A. and Galvan, M. . Fast inward-rectifying current accounts for anomalous rectification in olfactory cortex neurones, J. Physiol.. 335 (1983) 153-178.

4 Friedman. A. and Gutnick, M.J.. Low-threshold calcium electrogenesis in neocortical neurons. Neurosci. Lett.. 81 (1987) 117.122.

5 Hagiwara, S., Miyazaki, S., Moody, W. and Patlak, J., Blocking effects of barium and hydrogen ions on the potassium current during anomalous rectification in the starfish egg. J. Physiol.. 279 (1978) 167-185.

6 Hwa, G.G.C. and Avoli, M., Hyperpolarizing inward rectification in rat neocortical neurons located in the superficial layers. Neurosci. Lett., 124 (1981) 65-68.

7 Katz, B., Les constantes electriques de la membrane du muscle. Arch. Sci. Physiol., 3 (1949) 285-299.

8 Leech, C.A. and Stanfield, P.R., Inward rectification in frog skeletal muscle fibres and its dependence on membrane potential and external potassium, J. Physiol., 319 (1981) $295 \cdots 309$.

9 Mayer, M.L. and Westbrook, G.L., A voltage-clamp analysis of inward (anomalous) rectification in mouse spinal sensory ganglion neurones, J. Physiol., 340 (1983) 19-45.
10 McCormick. D.A. and Pape. H.-C.. Properties of a hyperpolarization-activated cation current and its role in thythmic oscillation in thalamic relay neurones. J. Physiol., 431 (1990) 291-318.

11 North. R.A., Drug receptors and the inhibition of nerve cells, Br. J. Pharmacol., 98 (1989) 13 28.

12 Schwindt. P.C. Spain, W., Foehring, R.C. Stafstrom, C.E., Chubb, M.C. and Crill, W.E., Multiple potassium conductances and their functions in neurons from cat sensorimotor cortex in vitro, J. Neurophysiol.. 59 (1988) 424449.

13 Spain, W.J., Schwindt, P.C. and Crill. W.E., Anomalous rectification in neurons from cat sensorimotor cortex in vitro, J. Neurophysiol., 57 (1987) 15551576.

14 Stafstrom. C.E., Schwindt. P.C.. Chubb, M.C. and Crill. W.E.. Properties of persistent sodium conductance and calcium conductance of layer $\mathrm{V}$ neurons from cat sensorimotor cortex in vitro, $\mathrm{J}$. Neurophysiol., 53 (1985) 153170.

15 Stafstrom. C.E., Schwindt. P.C., Flatman, J.A. and Crill, W.E., Properties of subthreshold response and action potential recorded in layer $\mathrm{V}$ neurons from cat sensorimotor cortex in vitro, J. Neurophysiol., 52 (1984) 244

16 Sutor. B. and Hablitz. J.J., Low concentrations of barium inhibit a potassium conductance contributing to the resting potential in rat neocortical neurons in vitro, Eur. J. Neurosci. Suppl., 5 (1992) 62.

17 Sutor, B. and Zieglgänsberger, W., A low-voltage activated, transient calcium current is responsible for the time-dependent depolarizing inward rectification of rat neocortical neurons in vitro, Pflügers Arch., 410 (1987) 102- 111 . 